(2) Open Access Full Text Article

REVIEW

\title{
Impact of "noncaloric" activity-related factors on the predisposition to obesity in children
}

This article was published in the following Dove Press journal:

Risk Management and Healthcare Policy

2 July 2010

Number of times this article has been viewed

\author{
Angelo Tremblay \\ Émilie Pérusse-Lachance \\ Patrice Brassard \\ Division de Kinésiologie, PEPS, \\ Université Laval and Centre de \\ Recherche de I'Institut Universitaire \\ en Cardiologie et Pneumologie de \\ Québec, Québec, Canada
}

\begin{abstract}
The research related to childhood obesity generally emphasizes the impact of unhealthy eating and sedentary behavior as the main determinants of the predisposition to the positive energy balance that underlies excess body fat accumulation. Recent investigations have, however, demonstrated that "noncaloric" activity-related factors can induce a significant imbalance between spontaneous energy intake and energy expenditure. This is the case for short sleep duration that favors hormonal changes that increase hunger and energy intake. This agrees with our research experience demonstrating that short sleeping predicts the risk of obesity in children to a greater extent than sedentary behavior. Recent research by our team has also showed that demanding mental work promotes a substantial increase in energy intake without altering energy expenditure. In addition, our preliminary data suggest that the regular practice of school-related cognitive efforts is predictive of an increase in abdominal fat accumulation. As discussed in this paper, individual variations in brain oxygenation and its related cerebral aerobic fitness might play a role in the relationship between mental work, energy intake, and the risk of excess body weight.
\end{abstract}

Keywords: sleep duration, mental work, brain oxygenation, energy intake, energy expenditure

\section{Introduction}

A successful preventive strategy of a health problem generally begins with the identification and the characterization of factors promoting the problem. With respect to obesity, it is known that the problem is the ultimate consequence of long term excess energy intake over expenditure. However, this principle, which reflects the implications of the first law of thermodynamics in the field of obesity, does not document the key issues pertaining to its determinants in a specific population. In this regard, the main question of interest refers to the factors that underlie a positive energy balance under free-living conditions and the habitual answer presented by health professionals is that unhealthy eating and sedentary behavior are explanatory factors.

As described in this paper, our recent research and that of others emphasize the relevance in considering activity-related factors which do not have a significant direct impact on energy expenditure, be it because of their low energy cost or their inability to increase energy expenditure when performed even at a high intensity. These factors include short sleeping and demanding mental work which can be viewed as "noncaloric" factors having the potential to induce a large caloric imbalance.
Correspondence: Angelo Tremblay Division de Kinésiologie, PEPS, Bureau 0234, Université Laval, Québec,

Canada GIV 0A6

$\mathrm{Tel}+\mathrm{I} 418-656-7294$

Fax +| 4|8-656-3044

Email angelo.tremblay@kin.msp.ulaval.ca 


\section{Short sleeping and obesity in children}

A careful review of the literature reveals that the apparently newly documented sleep-obesity relationship is not so new. For instance, at the beginning of the 1990s, a significant association was reported between sleep duration and body weight variations. ${ }^{1}$ However, this issue of investigation gained much credibility when the team of Van Cauter showed that short term experimentally controlled sleep deprivation induced a decrease in leptinemia and an increase in plasma ghrelin level. ${ }^{2}$ Accordingly, an increase in hunger sensation was observed in the sleep-restricted subjects. These observations as well as population data indicating a decrease in sleep duration over the last decades also led Van Cauter to propose an association between the progressive decrease in sleep duration and the development of an obesity epidemic. ${ }^{3}$

This research prompted us to incorporate sleep duration in the list of variables to be considered in analyses aimed at a better understanding of variations in body weight. In the Quebec en Forme Project, we examined the relationship between anthropometric indicators and various lifestyle markers known to predict the risk of obesity in children. As expected, we found that low education level and familial income, parental obesity, long television viewing, and sedentary behavior were all statistically significant predictors of an increased risk of obesity. ${ }^{4}$ However, we were surprised to note that short sleeping was by far the best predictor of obesity in this cohort. Further analyses also demonstrated that the excess body weight related to short sleep duration is preferentially deposited in the abdominal area in children. ${ }^{5}$ Considering that excess abdominal fat increases the risk to develop a metabolic syndrome in the long term, ${ }^{6}$ optimal sleep duration should be included in the indicators of lifestyle that are associated to optimal metabolic health.

We also took advantage of the Quebec Family Study to give extension to our analyses in an adult population. As in children, short sleepers were found to be heavier than normal sleepers reporting 7-8 $\mathrm{hr}$ sleep per day. ${ }^{7}$ In addition, this study showed that long sleepers were also displaying excess weight gain over time. ${ }^{8}$

The Quebec Family Study also includes some metabolic indicators which permit the investigation of some mechanistic explanations of the sleep-body fat relationship. We observed that short sleepers are characterized by a lower plasma leptin concentration than the level predicted by their body fat mass. ${ }^{\text {? }}$ We also noted that both short and long sleepers display an increased plasma glucose area below basal level during an oral glucose tolerance test. ${ }^{9}$ Since this hypoglycemic profile has been shown to predict long term weight gain, ${ }^{10}$ this may represent an explanation of the increased risk of obesity related to variations in sleep duration. Finally, it is relevant to emphasize that this increased proneness to mild hypoglycemia also represents an independent risk factor of glucose intolerance and diabetes. ${ }^{11}$

These observations suggest that sleep, which is the activity with the theoretically lowest energy cost, does not mainly influence body weight via its impact on energy expenditure. Its effects on body weight and fat seem to be mediated by metabolic effects that affect the control of appetite. This is concordant with the recent demonstration that sleep deprivation favors an increase in energy intake at snack time. ${ }^{12}$

\section{Mental work, energy intake, and body weight stability}

An environment that is increasingly globalized and relies on computerization to permit competitiveness inevitably requires additional cognitive effort. This is the case of workers in many fields of expertise and of children for whom learning activities become more demanding. In our opinion, this issue is not dissociated from the literature that documents the potential link between intellectual capacities and the proneness to obesity. As early as the 1960s, Canning and Mayer ${ }^{13}$ reported that the proportion of nonobese students in the upper quartile of academic success was greater than that of obese subjects. This issue was subsequently examined by Kreze et a ${ }^{14}$ who found a decreased obesity prevalence in women characterized by a high intellectual quotient (IQ). This trend was also observed in men but to a lesser extent than in women. This topic was further investigated by the group of Sorensen et a $\mathrm{l}^{15}$ who found lower results for intelligence tests in subjects characterized by a high body mass index (BMI). Accordingly, this team observed increased odd ratios of school difficulties in obese children. ${ }^{16}$ More recently, the same team concluded that intelligence tests and education level are negatively related with subsequent changes in BMI and the risk of obesity. ${ }^{17}$ This appears concordant with the results of Lawlor et a $1^{18}$ who also found a negative association between IQ at childhood and BMI at adulthood.

These studies and others have led to the very recent publication of a literature review pertaining to the theme of "intelligence and obesity" 19 which summarizes a gradient of opinion that, although not reflecting a consensus, might lead to the perception that IQ measurement would be a relevant tool of prediction of the proneness to obesity. We have reacted to the publication of this paper by proposing that demanding mental work can act as an intermediate factor in this relationship. ${ }^{20}$ 
The first study performed in our laboratory to investigate the metabolic effects of mental work was a case study in which one of us (AT) was subjected to either a 60-min cognitive effort to prepare a grant application or a relaxing session of similar duration. ${ }^{21}$ Unexpectedly, performing mental work promoted glycemic and insulinemic instability that was related to an increase in hunger and desire to eat. Moreover, concomitant experiments revealed that demanding knowledge-based work (KBW) increased spontaneous energy intake in graduate students. ${ }^{21}$

These preliminary studies were followed by investigations involving Laval University students who were randomly assigned to a reading-writing task or a relaxing session of $45 \mathrm{~min}$ each. In the first study, ${ }^{22}$ the use of indirect calorimetry showed that mental work exerts a trivial effect on energy expenditure since the total energy cost of the KBW session exceeded by only $3 \mathrm{kcal}$ the value measured during the control relaxing session. Conversely, the study showed that a large increase in ad libitum energy intake was observed after the cognitive effort (Study 1 in Table 1) and this was confirmed by the results of a subsequent study performed in another group of female university students (Study 2). ${ }^{23}$ Specifically, mental work induced a mean increase of $229 \mathrm{kcal}$ in energy intake during a buffet-type meal. In the second study, ${ }^{23}$ the reading-writing task promoted a comparable increase in ad libitum caloric intake. Furthermore, this spontaneous increase reached $250 \mathrm{kcal}$ when subjects of Study 2 performed a third session of computer tasks (Table 1).

The second study of Laval University students also gave us the opportunity to evaluate the effects of the intensity of mental work on energy intake and related variables. For that purpose, we measured the reaction time to a second task (vocal reaction by saying a short word in response to a randomly emitted noise) during the cognitive effort. As previously demonstrated, this method provides an indication of the intensity of mental effort which increases when reaction time increases and vice versa. ${ }^{24,25}$ In our hands, this technique also had a satisfactory discriminative potential since the greater was reaction time, ie, the greater the intensity of mental work, the greater was the increase in energy intake. ${ }^{26}$

The consideration of the effects of activity-related factors such as short sleep and mental work also led us to reconsider the notion of sedentary behavior which should no more be viewed as only reflecting the insufficiency of physical activity practice. We thus tested the predictive value of the risk of obesity in children based on the four following markers of sedentary activity: movement counts (accelerometry),
Table I Spontaneous energy intake ( $\mathrm{kJ}$ ) following rest and mental work

\begin{tabular}{llll}
\hline & Rest & Mental work & \\
\cline { 3 - 4 } & & Reading/writing & Computer games \\
\hline Study I & 3,923 & 4,882 & \\
Study 2 & 3,595 & 4,443 & 4,652 \\
\hline
\end{tabular}

Notes: See Chaput et a ${ }^{22,23}$ for Study I and 2, respectively.

television viewing, sleep duration, and time allocated to school homework. As expected, the suboptimal practice regarding each factor accentuated the risk to be overweight. ${ }^{27}$ Specifically, $64 \%$ of children were overweight/obese when their practice was suboptimal for each indicator of sedentary activity. Therefore, sedentary behavior appears as a cluster of activities which could influence energy balance via different mechanisms and according to different temporal modalities. For instance, a low movement count is related to a greater risk of obesity whereas a low duration of sleep predicts the opposite effect on body weight.

\section{Towards the characterization of cerebral aerobic fitness}

The brain needs uninterrupted oxygen provision and delivery of oxygen to cerebral tissue is mainly provided by its binding to hemoglobin. The evaluation of cerebral hemoglobin oxygenation represents the balance between changes in blood flow through the tissue and cerebral oxygen consumption. ${ }^{28}$ A high energy demand is mainly met through oxidative phosphorylation in the brain mitochondria, ${ }^{29,30}$ which is responsible at rest for the consumption of most glucose used by the brain. This process is closely coupled to oxygen utilization of the brain and is responsible for about $20 \%$ of total oxygen consumption in the human body. ${ }^{31}$ Mental work is associated with changes in cerebral oxygenation which has been shown by the use of near-infrared spectroscopy (NIRS). For instance, frontal lobe oxygenation increases with cognitive processing using a measure of verbal fluency, ${ }^{32}$ lateral frontal lobe oxygenation elevates with translation of short sentences and language switching in bilingual student volunteers, ${ }^{33}$ and oxygenation of the inferior and superior parietal areas increase with processing and solving arithmetic problems. ${ }^{34}$ Specifically, the NIRS-derived frontal lobe concentration of oxygenated hemoglobin increases and the concentration of deoxygenated hemoglobin decreases when performing cognitive tasks. ${ }^{35}$ This may be associated with an increase or the absence of a change in total hemoglobin concentration. ${ }^{36}$ Such changes in cerebral oxygenation measured by positron emission tomography are considered to represent 
cerebral activation, ${ }^{37}$ and a good agreement exists between measurements of cerebral oxygenation derived from NIRS and functional magnetic resonance in young and elderly subjects during brain activation. ${ }^{38}$ Importantly, the difficulty level of a mental task, such as an algorithm task, increases NIRS-derived cerebral oxygenation. ${ }^{34-36}$ The discussion of these issues is also relevant for the recovery of a mental task during which brain activation remains elevated. ${ }^{39}$ Although a fraction of this elevated brain activation may be related to mental processing, it could also be attributed to metabolic and other neuronal functions. Since frontal lobes are involved in functions such as planning, organization, problem solving, memory, and judgment, it is important to examine the hypothesis according to which brain oxygenation represents a determinant of the intensity of mental work and its related metabolic changes.

The more pronounced increase in NIRS-derived frontal lobe oxygenation potentially characterizing individuals who perceive a mental task as difficult has a certain number of nutritional implications. As discussed above, the effect on which our research team has mostly focused on up to now is a change in spontaneous energy intake that was repeatedly found to be increased by 200 to $250 \mathrm{kcal}$ following demanding mental work. ${ }^{21,22,40}$ This observation is concordant with the results of Wallis et $\mathrm{al}^{41}$ who reported that chocolate intake was increased by $15 \%$ after a stressful cognitive task (Stroop Test) compared to a control session.

There are also indications that the increased energy intake resulting from mental work is related to variations in cortisolemia. Indeed, we have demonstrated that female university students perceiving a standardized mental task as more demanding also displayed a more pronounced increase in energy intake and cortisolemia. ${ }^{42}$ Accordingly, the study of Epel et $\mathrm{al}^{43}$ showed that stress-induced cortisol reactivity was associated with an increased energy intake after the first stress session. Since cortisol is well known for its orexigenic properties, it seems logical to hypothesize that the greater the increase in cortisolemia induced by mental work, the greater its hyperphagic effect. We also believe that spontaneous energy intake will be increased in these subjects.

According to the glucostatic theory of appetite control, ${ }^{44}$ the hyperphagia induced by mental work can be reasonably associated with variations in the availability of glucose. Indeed, since neurons essentially rely on glucose for their metabolism under normal feeding conditions and that cerebral carbohydrate stores are low, it is likely that the increased brain energy metabolism imposed by a cognitive effort implies an equivalent increase in glucose oxidation. In this regard, the data recently reported by Volkow et $\mathrm{al}^{45}$ are particularly informative. They indeed showed that the amount of glucose utilized by the brain is increased when performing a cognitive task. They also demonstrated that methylphenidate, which is known for its properties to stimulate brain functioning and thus reducing attention deficit, attenuated the increase in brain glucose oxidation when performing the cognitive task. Such an effect was not observed when there was no cognitive stimulation. In our opinion, these observations are quite compatible with the main concept presented here, ie, decreased difficulty of a cognitive task reflects a metabolically efficient process with a reduced metabolic demand. We also perceive these results as being in accordance with the lower instability of plasma glucose concentrations that we observed in female university students for whom the estimated intensity of mental work was reduced. ${ }^{26}$ This is also in agreement with a recent case study that we performed by using methylphenidate to reduce the stress of the cognitive effort. Indeed, blood pressure was then decreased during the mental work that was followed by a diminished spontaneous caloric intake (Pérusse-Lachance et al in preparation). Taken together, these observations suggest once again that a greater mental effort is metabolically disturbing, probably via modifications in glucose metabolism.

Another category of nutritional variables that is worth considering in the study of the mental work-brain oxygenation-obesity relationship is hematological profile. To our knowledge, Wenzel et $\mathrm{al}^{46}$ were the first investigators to note a relationship between obesity and anemia. In 1962, they reported that obese adolescents had a lower serum iron level than adolescents of normal weight. Concordant results were further reported by Seltzer and Mayer. ${ }^{47}$ More recently, it has been possible to confirm and quantify the association between body weight and anemia since three cross-sectional studies documented the prevalence of iron deficiency in children and adolescents of different body weights. In each study, it appears that the iron deficit was greater in overweight or obese children and adolescents than in normal weight individuals. ${ }^{48-50}$ Obesity seems to have a similar effect on iron status in adults. Indeed, Micozzi et $\mathrm{al}^{51}$ found that an increase in body weight in 25 to 74 year-old women was associated with a decrease in serum iron concentrations. In obese post-menopausal women, similar results were reported, as reflected by a positive correlation between BMI and soluble transferrin receptor levels. ${ }^{52}$ With respect to the specific link between the hematological profile and the cognitive function, the most relevant data were reported by Krestch et $\mathrm{al}^{53}$ who observed a reducing effect of weight loss on hemoglobin, 
hematocrit, and red blood count. Interestingly, they also noted a significant association between this change in the hematological profile and the decrease in the cognitive function of their subjects. In summary, these findings emphasize the hematological vulnerability of obesity-prone individuals and the possibility that such a profile be part of the etiology of the association between mental work and obesity that is discussed in this paper.

The global integration of the above discussed literature tends to show that a good "cerebral aerobic fitness" would permit performance of a given mental task with a reduced brain activation reflected by a lower NIRS-derived frontal lobe oxygenation. As discussed above, this greater efficiency is likely associated with a better metabolic fitness, be it reflected by a more stable glycemia, a reduced cortisolemia, a facilitated appetite control, and a lower blood pressure.

\section{Conclusion}

This paper summarizes our research experience regarding the effects of short sleeping and demanding mental work as activity modalities which can influence body composition. Since their energy cost is very low, it is not surprising to observe that their potential effects on energy balance are mediated by a significant impact on energy intake and related metabolic variables. Thus, the consideration of these "noncaloric" factors as determinants of the risk of obesity impose on us to review the perception of sedentary behavior which is not simply an insufficient physical activity practice. Furthermore, this paper reminds us that we have entered a new era where cerebral fitness will be an issue, be for its link with cognitive performance and productivity or its side effects such as the hyperphagia induced by mental work.

\section{Disclosure}

The authors report no conflicts of interest in this work.

\section{References}

1. Locard E, Mamelle N, Billette A, Miginiac M, Munoz F, Rey S. Risk factors of obesity in a five year old population. Parental versus environmental factors. Int J Obes Relat Metab Disord. 1992;16(10): 721-729.

2. Spiegel K, Tasali E, Penev P, Van Cauter E. Brief communication: sleep curtailment in healthy young men is associated with decreased leptin levels, elevated ghrelin levels, and increased hunger and appetite. Ann Intern Med. 2004;141(11):846-850.

3. Van Cauter E, Spiegel K, Tasali E, Leproult R. Metabolic consequences of sleep and sleep loss. Sleep Med. 2008;9 Suppl 1:S23-S28.

4. Chaput JP, Brunet M, Tremblay A. Relationship between short sleeping hours and childhood overweight/obesity: results from the 'Quebec en Forme' Project. Int J Obes (Lond). 2006;30(7):1080-1085.

5. Chaput JP, Tremblay A. Does short sleep duration favor abdominal adiposity in children? Int J Pediatr Obes. 2007;2(3):188-191.
6. Despres JP, Lemieux I, Prud'homme D. Treatment of obesity: need to focus on high risk abdominally obese patients. $B M J$. 2001;322(7288):716-720.

7. Chaput JP, Despres JP, Bouchard C, Tremblay A. Short sleep duration is associated with reduced leptin levels and increased adiposity: results from the Quebec family study. Obesity (Silver Spring). 2007;15(1):253-261.

8. Chaput JP, Despres JP, Bouchard C, Tremblay A. The association between sleep duration and weight gain in adults: a 6-year prospective study from the Quebec Family Study. Sleep. 2008;31(4):517-523.

9. Chaput JP, Despres JP, Bouchard C, Tremblay A. Association of sleep duration with type 2 diabetes and impaired glucose tolerance. Diabetologia. 2007;50(11):2298-2304.

10. Boule NG, Chaput JP, Doucet E, et al. Glucose homeostasis predicts weight gain: prospective and clinical evidence. Diabetes Metab Res Rev. 2008;24(2):123-129.

11. Chaput JP, Despres JP, Bouchard C, Astrup A, Tremblay A. Sleep duration as a risk factor for the development of type 2 diabetes or impaired glucose tolerance: analyses of the Quebec Family Study. Sleep Med. 2009;10(8):919-924.

12. Nedeltcheva AV, Kilkus JM, Imperial J, Kasza K, Schoeller DA, Penev PD. Sleep curtailment is accompanied by increased intake of calories from snacks. Am J Clin Nutr. 2009;89(1):126-133.

13. Canning H, Mayer J. Obesity: an influence on high school performance? Am J Clin Nutr. 1967;20(4):352-354.

14. Kreze A, Zelina M, Juhas J, Garbara M. Relationship between intelligence and relative prevalence of obesity. Hum Biol. 1974;46(1):109-113.

15. Sorensen TI, Sonne-Holm S, Christensen U, Kreiner S. Reduced intellectual performance in extreme overweight. Hum Biol. 1982;54(4):765-775.

16. Lissau I, Sorensen TI. School difficulties in childhood and risk of overweight and obesity in young adulthood: a ten year prospective population study. Int J Obes Relat Metab Disord. 1993;17(3):169-175.

17. Halkjaer J, Holst C, Sorensen TI. Intelligence test score and educational level in relation to BMI changes and obesity. Obes Res. 2003;11(10):1238-1245.

18. Lawlor DA, Clark H, Davey Smith G, Leon DA. Childhood intelligence, educational attainment, and adult body mass index: findings from a prospective cohort and within sibling-pairs analysis. Int J Obes (Lond). 2006;30(12):1758-1765.

19. Yu ZB, Han SP, Cao XG, Guo XR. Intelligence in relation to obesity: a systematic review and meta-analysis. Obes Rev. 2009 Sep 23. [Epub ahead of print]

20. Chaput JP, Tremblay A. Intelligence and obesity: does the intensity of mental workload matter? Obes Rev. 2010 Feb 8. [Epub ahead of print]

21. Tremblay A, Therrien F. Physical activity and body functionality: implications for obesity prevention and treatment. Can J Physiol Pharmacol. 2006;84(2):149-156.

22. Chaput JP, Tremblay A. Acute effects of knowledge-based work on feeding behavior and energy intake. Physiol Behav. 2007;90(1):66-72.

23. Chaput JP, Drapeau V, Poirier P, Teasdale N, Tremblay A. Glycemic instability and spontaneous energy intake: association with knowledgebased work. Psychosom Med. 2008;70(7):797-804.

24. Humphrey DG, Kramer AF. Toward a psychophysiological assessment of dynamic changes in mental workload. Hum Factors. 1994;36(1):3-26.

25. Ullsperger P, Freude G, Erdmann U. Auditory probe sensitivity to mental workload changes - an event-related potential study. Int $J$ Psychophysiol. 2001;40(3):201-209.

26. Chaput JP, Tremblay A. The glucostatic theory of appetite control and the risk of obesity and diabetes. Int J Obes (Lond). 2009;33(1):46-53.

27. Mathieu ME, Chaput JP, O’Loughlin J, Lambert M, Tremblay A. Current guidelines may protect children against overweight and abdominal obesity. Obes Facts. 2009;2:72.

28. Teng Y, Ding H, Gong Q. Non-invasive monitoring of human cerebral oxygen saturation by near infrared spectroscopy: instrumentation, calibration, and application in cardiopulmonary bypass. Conf Proc IEEE Eng Med Biol Soc. 2005;1:670-673. 
29. Attwell D, Laughlin SB. An energy budget for signaling in the grey matter of the brain. J Cereb Blood Flow Metab. 2001;21(10):1133-1145.

30. Du F, Zhu XH, Zhang Y, et al. Tightly coupled brain activity and cerebral ATP metabolic rate. Proc Natl Acad Sci U S A. 2008;105(17): 6409-6414.

31. Zhu XH, Zhang N, Zhang Y, Ugurbil K, Chen W. New insights into central roles of cerebral oxygen metabolism in the resting and stimulusevoked brain. J Cereb Blood Flow Metab. 2009;29(1):10-18.

32. Jayakar A, Dunoyer C, Rey G, Yaylali I, Jayakar P. Near-infrared spectroscopy to define cognitive frontal lobe functions. $J$ Clin Neurophysiol. 2005;22(6):415-417.

33. Quaresima V, Ferrari M, van der Sluijs MC, Menssen J, Colier WN. Lateral frontal cortex oxygenation changes during translation and language switching revealed by non-invasive near-infrared multi-point measurements. Brain Res Bull. 2002;59(3):235-243.

34. Richter MM, Zierhut KC, Dresler T, et al. Changes in cortical blood oxygenation during arithmetical tasks measured by near-infrared spectroscopy. J Neural Transm. 2009;116(3):267-273.

35. Villringer A, Planck J, Hock C, Schleinkofer L, Dirnagl U. Near infrared spectroscopy (NIRS): a new tool to study hemodynamic changes during activation of brain function in human adults. Neurosci Lett. 1993;154(1-2):101-104.

36. Hoshi Y, Tamura M. Detection of dynamic changes in cerebral oxygenation coupled to neuronal function during mental work in man. Neurosci Lett. 1993;150(1):5-8.

37. Fox PT, Raichle ME. Focal physiological uncoupling of cerebral blood flow and oxidative metabolism during somatosensory stimulation in human subjects. Proc Natl Acad Sci U SA. 1986;83(4):1140-1144.

38. Mehagnoul-Schipper DJ, van der Kallen BF, Colier WN, et al. Simultaneous measurements of cerebral oxygenation changes during brain activation by near-infrared spectroscopy and functional magnetic resonance imaging in healthy young and elderly subjects. Hum Brain Mapp. 2002;16(1):14-23.

39. Lichty W, Sakatania K, Lin F, Sun P, Ding H, Wang F. Near-infrared spectroscopic investigations of oxygenation changes related to brain activation. Proc SPIE. 1999;3863:197-201.

40. Chaput JP, Arguin H, Gagnon C, Tremblay A. Increase in depression symptoms with weight loss: association with glucose homeostasis and thyroid function. Appl Physiol Nutr Metab. 2008;33(1):86-92.
41. Wallis DJ, Hetherington MM. Stress and eating: the effects of ego-threat and cognitive demand on food intake in restrained and emotional eaters. Appetite. 2004;43(1):39-46.

42. Chaput JP, Tremblay A. Obesity and physical inactivity: the relevance of reconsidering the notion of sedentariness. Obes Facts. 2009;2(4):249-254.

43. Epel E, Lapidus R, McEwen B, Brownell K. Stress may add bite to appetite in women: a laboratory study of stress-induced cortisol and eating behavior. Psychoneuroendocrinology. 2001;26(1):37-49.

44. Mayer J. Glucostatic mechanism of regulation of food intake. $N$ Engl J Med. 1953;249(1):13-16.

45. Volkow ND, Fowler JS, Wang GJ, et al. Methylphenidate decreased the amount of glucose needed by the brain to perform a cognitive task. PLoS One. 2008;3(4):e2017.

46. Wenzel BJ, Stults HB, Mayer J. Hypoferremia in obese adolescents. Lancet. 1962;2(7251):327-328.

47. Seltzer CC, Mayer J. Serum iron and iron-binding capacity in adolescents. II. Comparison of obese and non-obese subjects. Am J Clin Nutr. 1963;13:354-361.

48. Nead KG, Halterman JS, Kaczorowski JM, Auinger P, Weitzman M. Overweight children and adolescents: a risk group for iron deficiency. Pediatrics. 2004;114(1):104-108.

49. Pinhas-Hamiel O, Newfield RS, Koren I, Agmon A, Lilos P, Phillip M. Greater prevalence of iron deficiency in overweight and obese children and adolescents. Int J Obes Relat Metab Disord. 2003;27(3): 416-418.

50. Tussing-Humphreys LM, Liang H, Nemeth E, Freels S, Braunschweig CA. Excess adiposity, inflammation, and iron-deficiency in female adolescents. J Am Diet Assoc. 2009;109(2):297-302.

51. Micozzi MS, Albanes D, Stevens RG. Relation of body size and composition to clinical biochemical and hematologic indices in US men and women. Am J Clin Nutr. 1989;50(6):1276-1281.

52. Lecube A, Carrera A, Losada E, Hernandez C, Simo R, Mesa J. Iron deficiency in obese postmenopausal women. Obesity (Silver Spring). 2006;14(10):1724-1730.

53. Kretsch MJ, Green MW, Fong AK, Elliman NA, Johnson HL. Cognitive effects of a long-term weight reducing diet. Int $J$ Obes Relat Metab Disord. 1997;21(1):14-21.
Risk Management and Healthcare Policy

\section{Publish your work in this journal}

Risk Management and Healthcare Policy is an international, peerreviewed, open access journal focusing on all aspects of public health, policy, and preventative measures to promote good health and improve morbidity and mortality in the population. The journal welcomes submitted papers covering original research, basic science, clinical \& epidemio-

\section{Dovepress}

logical studies, reviews and evaluations, guidelines, expert opinion and commentary, case reports and extended reports. The manuscript management system is completely online and includes a very quick and fair peerreview system, which is all easy to use. Visit http://www.dovepress.com/ testimonials.php to read real quotes from published authors. 